\title{
Mycophenolate induced GI mucosal injury - case report and review of the literature
}

\section{Introduction}

Mycophenolate mofetil (MMF) is an immunosuppressant widely used for the prevention of organ transplant rejection. It inhibits inosine monophosphate dehydrogenase (IMPDH), a key enzyme in de novo purine biosynthesis. As $90 \%$ of lymphocytes are dependent on this pathway, MMF is effective at suppressing lymphocyte proliferation and thus acute organ rejection. Since its introduction in the 1990's, it has significantly reduced the incidence of acute rejection in solid organ transplant recipients and is increasingly utilized as a corticosteroidsparing treatment in autoimmune disorders. However, gastrointestinal (GI) side effects remain very common, including abdominal pain, nausea, vomiting and diarrhea. While these symptoms often occur without any evidence of direct mucosal damage, several studies have demonstrated evidence of MMF-induced injury in both the upper and lower GI tract. Here we report a rare case of circumferential esophagitis and symptomatic esophageal stricture secondary to MMF.

\section{Case}

A 46 year-old male with end stage renal disease underwent deceased donor kidney transplant followed by immunosuppression with MMF, tacrolimus, and prednisone. Shortly after transplantation, he noted new solid food intermittent dysphagia with slow progression over 6 months. He was later hospitalized with progressive worsening of nausea, vomiting and dysphagia. Esophagogastroduodenoscopy (EGD) showed LA Class D esophagitis about $10 \mathrm{~cm}$ above the gastroesophageal junction with a stenosisat $30 \mathrm{~cm}$ from the incisors. Biopsies showed acute and chronic inflammation without increased eosinophils or evidence of Barrett's mucosa. Stains for fungi, cytomegalovirus (CMV) and herpesvirus (HSV) were negative. He was treated with esomeprazole $40 \mathrm{mg}$ twice a day but was re-hospitalized one month later with persistent symptoms. A repeat EGD demonstrated once again LA Class D esophagitis with worsening stricture in the proximal part of the lower third of the esophagus along with candida plaques. Biopsies revealed severe esophagitis without CMV/HSV (Figure 1). The stricture was dilated up to 44 Fusing Maloney dilators and fluconazole and nystatin were added to his proton pump inhibitor (PPI).

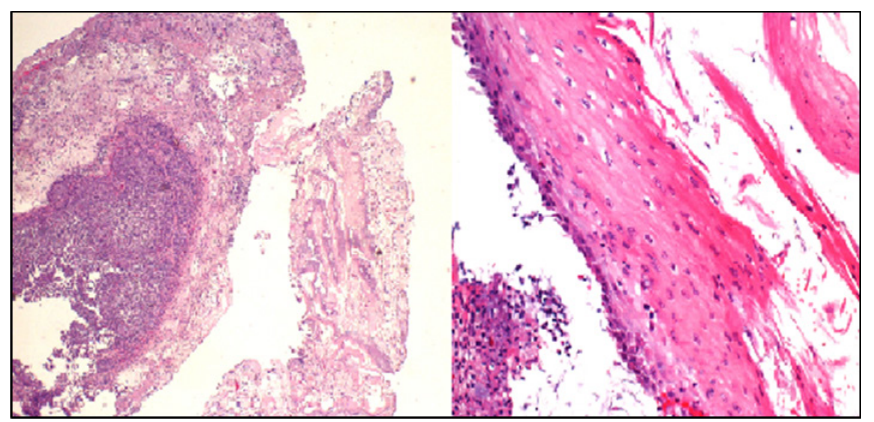

Figure I 20x (left) and 40x (right) views of desquamated squamous epithelium with associated acute fibrino-inflammatory infiltrates consisting predominantly of neutrophils with background reactive cytologic changes.

\author{
Volume 8 Issue 3 - 2018 \\ Tasnia Matin, Yan Zhao, Jatinder Goyal, \\ Frederick Weber \\ University of Alabama at Birmingham, USA
}

Correspondence: Tasnia Matin, University of Alabama at Birmingham, USA, Tel 3346639730 , Email tasniamatin@uabmc.edu

Received: March 27, 2018| Published: May 02, 2018

Symptoms recurred despite compliance with therapy and EGD two months later showed persistent LA Class D esophagitis without histologic evidence of opportunistic infection. A bariums wallow showed a tight $3 \mathrm{~cm}$ long, $4 \mathrm{~mm}$ diameter stricture in the same location (Figure 2). Given the unusually refractory nature and midesophageal location of his circumferential esophagitis and stricture and no histologic evidence of Barrett's, opportunists, or neoplasia, MMF was suspected to be the culprit. His MMF was discontinued and azathioprine was substituted. He was re-dilated to $12 \mathrm{~mm}$ using a through the scope (TTS) balloon. Barium swallow (Figure 3) at 3-month follow-up showed no evidence of esophagitis or stricture and PPI therapy was discontinued without symptomatic recurrence. At 2.5 year follow up he continues to be symptom free off of PPI therapy.

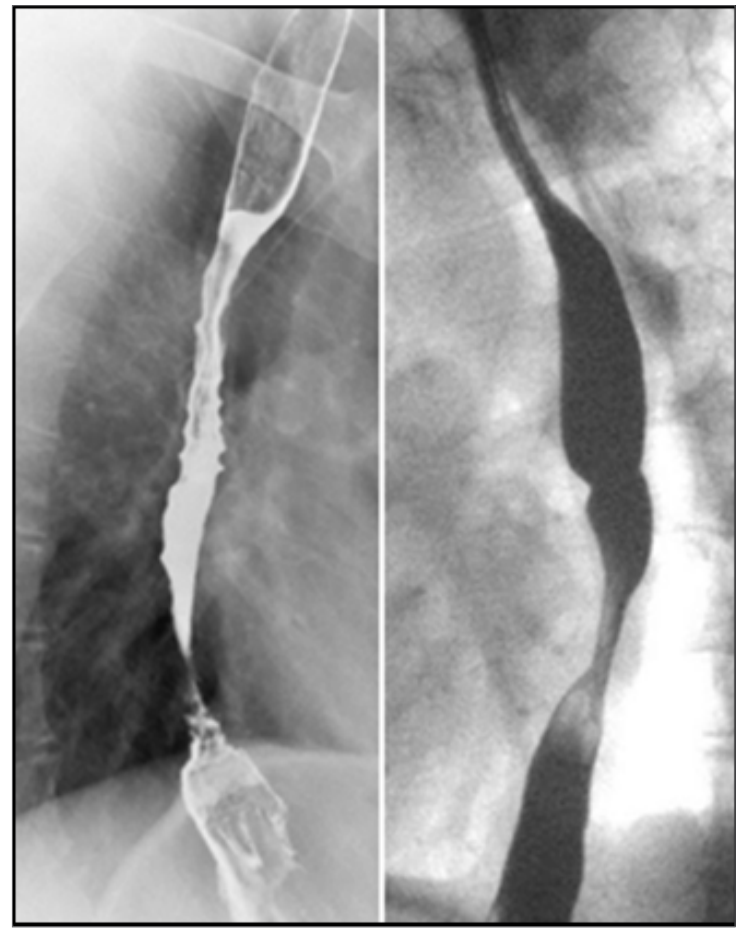

Figure 2 Barium swallow in November 2013 showed a tight $3 \mathrm{~cm}$ long, $4 \mathrm{~mm}$ diameter stricture in the lower third of the esophagus. 


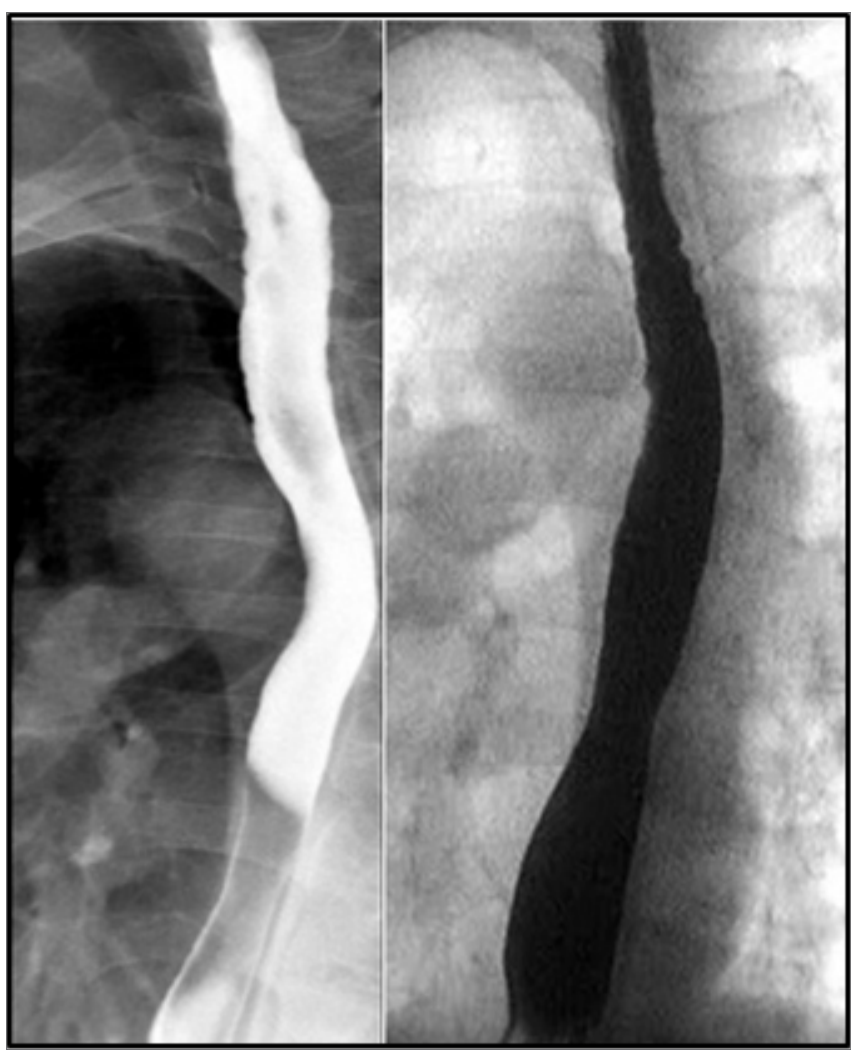

Figure 3 Normal barium swallow at 3 month follow-up.

\section{Discussion}

MMF is an immunosuppressant commonly used for the prevention of acute allograft rejection in solid organ transplant patients. It is a prodrug of mycophenolic acid, which reversibly inhibits inosine monophosphate dehydrogenase (IMPDH) in the de novo synthesis of purines. ${ }^{1}$ As $\mathrm{T}$ and B-lymphocytes require purines for DNA synthesis, MMF suppresses lymphocytic proliferation and thus impairs the body's ability to mount an immune response against transplant allografts. MMF has well-known gastrointestinal adverse effects, including abdominal pain, nausea, vomiting and diarrhea. The exact mechanism is unknown, but the vulnerability of the GI tract is most likely a result of the dependence of enterocytes on the de novo synthetic pathway of purines, which is disrupted by MMF. ${ }^{2}$ While the entire GI tract is at increased risk of MMF toxicity, the lower GI tract, namely the colon and less frequently the small bowel, appear to have been disproportionately affected. In75 GI biopsies from 46 transplant recipients, Jeremy et al showed pathologic features throughout the GI tract due to MMF. Among the 16 patients on MMF who underwent colonoscopy, a variety of mucosal injuries were observed in the lower GI tract, ranging from IBD-like changes in $13 \%$ of the biopsies to increased lamina propria inflammation in $81 \%$. Graft-versus-hostdisease (GVHD)-like features, including mild crypt architectural disarray, villous blunting, edema, and increased crypt epithelial apoptosis, was observed in $56 \%$ of the biopsies. ${ }^{3,4}$ Moreover, the presence of eosinophils within the lamina propria, endocrine cell aggregates, and hypereosinophilic crypts were morphologic features shown to be independently associated with MMF-induced colitis. ${ }^{5}$

Whereas middle and lower GI tract injuries may reflect the antimetabolite activity of MMF, resulting in GVHD-like changes such as colitis; the upper GI mucosal injuries may reflect local mucosal irritation, giving rise to erosive esophagitis (EE), reactive gastropathytype changes and/orduodenal ulcers. ${ }^{4}$ Soo Kim et al. ${ }^{6}$ showed a significant correlation between incidence of erosive esophagitis and MMF therapy in solid transplant recipients. Furthermore, Parfitt et al. ${ }^{4}$ documented that out of 7 esophageal biopsies from patients on MMF, 5 displayed variable degrees of active esophagitis. Histologic abnormalities in biopsies were also observed in the stomach and small bowel, ranging from $40-60 \%{ }^{3}$ In the stomach, moderate-tosevere gastropathy was observed in 4 of the 10 biopsies from patients receiving MMF. One gastric biopsy also showed Crohn's-like features, including granulomatous inflammation, which was also observed on duodenal and ileal biopsy. In addition, 6 of the 17 small bowel (duodenum, ileum) biopsies from patients on MMF showed GVHDlike features. ${ }^{4}$ These injuries tend to occur early, usually within the first three to twelve months of initiating MMF therapy. ${ }^{6}$ The risk of gastroduodenal erosions was approximately 1.83 fold for MMF, with the highest risk of erosive lesions associated with MMF-tacrolimuscorticosteroid combination treatment. ${ }^{6-8}$

While it is important to consider MMF as a possible cause of esophageal injury in a post-transplant patient, one must first consider other more common causes, including reflux esophagitis, pill esophagitis, infectious esophagitis (HSV, CMV, and candida), radiation esophagitis, and corrosive ingestion. In the middle and lower GI tract, the differential diagnosis should also include inflammatory bowel disease, infections (bacterial, viral, and parasitic), microscopic colitis, malabsorption, post-transplant immunoproliferative disease, and ischemia. Once MMF is confirmed as the cause of mucosal injury, the goal is to reduce or eliminate MMF from the immunosuppressive regimen.

Despite the increasing recognition of MMF-induced GI tract mucosal injury, we are reporting the first clinical case of circumferential esophagitis with stricture of which we are aware. The pathologic changes observed included inflamed, necrotic squamous cells, fibrinoinflammatory exudates and fibrin deposits in the ulcer bed, but unlike many patients with small bowel or colonic damage from MMF, they did not have the mucosal features often characteristic of GVHD. Cessation of mycophenolate and dilation of the esophageal stricture led to complete clinical, radiographic, and endoscopic resolution. Moreover, the lack of symptomatic recurrence in the absence of PPI therapy for the following several years further supports MMF-induced esophageal injury as the etiology. In conclusion, a diagnosis of MMFinduced esophagitis, while rare, should be considered in a patient with dysphagia when more common etiologies have been excluded or when esophagitis is PPI refractory. Pathologic findings include desquamated squamous epithelium with fibrino-inflammatory exudates, granulation tissue, and neutrophilic infiltrates. Unlike small bowel and colonic mucosal injury from MMF, microscopic changes may not reflect findings similar to GVHD. MMF-related esophageal mucosal injury resolves with discontinuation of the immunosuppressant.

\section{Acknowledgements}

None. 


\section{Conflict of interest}

The author declares there is no conflict of interest.

\section{References}

1. Fulton B, Markham A. Mycophenolate mofetil: a review of its pharmacodynamic and pharmacokinetic properties and clinical efficacy in renal transplantation. Drugs. 1996;51(2):278-98.

2. Behrend M. Adverse gastrointestinal effects of mycophenolate mofetil: aetiology, incidence and management. Drug Safety. 2001;24(9):645-63.

3. Al-Absi AI, Cooke CR, Wall BM, et al. Patterns of injury in mycophenolate mofetil-related colitis. Transplant Proc. 2010;42(9): 3591-3.

4. Parfitt JR, Jayakumar S, Driman DK. Mycophenolate mofetil-related gastrointestinal mucosal injury: variable injury patterns, including graftversus-host disease-like changes. Am J Surg Pathol. 2008;32(9):1367-72.
5. Star KV, Ho VT, Wang HH, et al. Histologic features in colon biopsies can discriminate mycophenolate from GVHD-induced colitis. Am J Surg Pathol. 2013;37(9):1319-28.

6. Kim IS, Lee H, Park JC, et al. Increased incidence of endoscopic erosive esophagitis in solid organ transplant recipients. Gut and liver. 2012;6(3):349-54.

7. Telkes G, Peter A, Tulassay Z, et al. High frequency of ulcers, not associated with Helicobacter pylori, in the stomach in the first year after kidney transplantation. Nephrol Dial Transplant. 2011;26(2):727-32.

8. Nguyen T, Park JY, Scudiere JR, et al. Mycophenolic acid (Cellcept and Myfortic) induced injury of the upper GI tract. Am J Surg Pathol. 2009;33(9):1355-63. 